Case

Report

\title{
Coronary Artery Bypass Grafting in a Patient with Polyarteritis Nodosa Presenting with Acute Myocardial Infarction and Multiple Coronary Aneurysms
}

Hidetake Kawajiri, MD, Eisei Koh, MD, Noriyasu Masuda, MD, Hiromasa Kira, MD, and Takuma Yamasaki, MD

\begin{abstract}
Polyarteritis nodosa (PAN) is a necrotizing form of vasculitis that affects small- and mediumsized vessels. Cases of ischemic heart disease involving coronary aneurysms in patients with PAN have been reported previously, but there have only been a few reports of coronary artery surgery for PAN-related coronary disease. A 46-year-old female with a history of PAN arrived at our emergency room due to cardiopulmonary arrest. After cardiopulmonary resuscitation, emergent coronary angiography was performed, because an electrocardiogram demonstrated ST segment elevation in leads V1 to V5. Coronary angiography revealed occlusion of the proximal right coronary artery and left anterior descending coronary artery as well as multiple coronary aneurysms. We performed emergent two-vessel coronary artery bypass grafting (the left anterior descending coronary artery and posterolateral branch of the circumflex system were treated with grafts from the left internal mammary artery and saphenous vein graft, respectively) under percutaneous cardiopulmonary support without cardiac arrest. The patient's postoperative course was uneventful, and postoperative coronary angiography revealed that the bypass grafts were patent. A review of the literature and a discussion of this case are also presented.
\end{abstract}

Keywords: coronary artery bypass grafting, polyarteritis nodosa

\section{Introduction}

Polyarteritis nodosa (PAN) is a multi-organ necrotizing form of vasculitis that affects small and medium sized vessels and is commonly seen in the kidneys, gastrointestinal tract, skin, and muscles. In a previous report, the incidence of PAN was reported to be 2.4 per million. ${ }^{1)}$

Department of Cardiovascular Surgery, Kyoto Second Red Cross Hospital, Kamigyo-ku, Kyoto, Japan

Received: January 31, 2013; Accepted: May 29, 2013

Corresponding author: Hidetake Kawajiri, MD. Department of

Cardiovascular Surgery, Kyoto Second Red Cross Hospital, 355-5

Haruobi-Cho, Kamigyo-ku, Kyoto 602-8026, Japan

Email: kawajiricvs@yahoo.co.jp

(C)2014 The Editorial Committee of Annals of Thoracic and Car-

diovascular Surgery. All rights reserved.
Hepatitis B virus infection is the only environmental factor that is known to be associated with PAN. ${ }^{2}$ Vasculitis in the coronary arteries can lead to vessel occlusion and aneurysmal dilatation, but is unusual. Herein, we report a case of PAN combined with acute myocardial infarction involving multiple coronary aneurysms in which the patient underwent emergent coronary artery bypass grafting.

\section{Case Report}

A 46-year-old woman with a history of PAN was found in a state of cardiopulmonary arrest. The electrocardiogram recorded by the automated external defibrillator revealed ventricular fibrillation. She was diagnosed with PAN 12 years ago and had been prescribed medication 


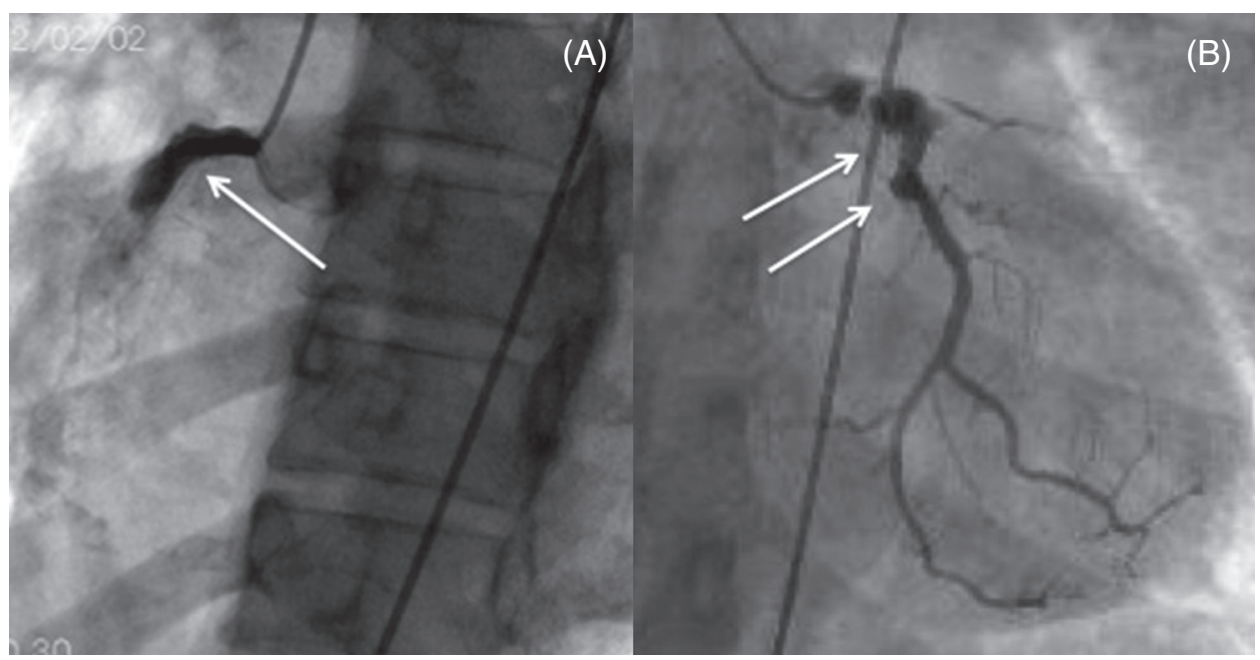

Fig. 1 Preoperative coronary angiography showing occlusion of the right coronary artery (A) and the proximal segment of the left anterior descending coronary artery (B) multiple coronary aneurysms (arrows).

including oral prednisone and cyclophosphamide, but she stopped taking the medication within a few years. She had no cardiac risk factors, such as diabetes, hyperlipidemia, smoking and had never been infected with hepatitis B virus. Cardiopulmonary support was continued, and she was taken to the emergency room. An electrocardiogram showed pulseless electrical activity. After tracheal intubation and the intravenous administration of adrenalin, her electrocardiogram recovered to sinus rhythm, and her systolic blood pressure was elevated to $80 \mathrm{mmHg}$. An electrocardiogram exhibited ST segment elevation in leads V1 to V5, which was suggestive of acute myocardial infarction of the anterior wall, and emergent coronary angiography was performed. Coronary angiography revealed occlusion of the proximal right coronary artery and left anterior ascending coronary artery, as well as multiple coronary aneurysms (Fig. 1). During coronary angiography, percutaneous cardiopulmonary support was initiated, and an intra-aortic balloon pump was inserted because of the patient's hemodynamic instability. Percutaneous coronary intervention was attempted, but it was unsuccessful and so emergent coronary artery bypass grafting was performed.

In this procedure, an ultrasonically skeletonized left internal mammary artery (in situ LIMA) was used to replace the left anterior descending coronary artery, and a saphenous vein graft was used to replace the posterolateral branch of the circumflex system. Proximal anastomosis of the saphenous vein graft was achieved using the PAS-Port Proximal Anastomosis System (Cardica, Inc.,
Redwood City, California, USA). Severe calcification and ectasia were observed in a proximal lesion in the right coronary artery, but no distal lesions were found to be suitable for anastomosis. The whole procedure was completed under percutaneous cardiopulmonary support, without cardiac arrest.

The patient had no neurological complications and was weaned off the percutaneous cardiopulmonary support on postoperative day 1. Her postoperative course was unremarkable, and she was discharged from hospital 2 weeks later.

We histopathologically re-examined the lung biopsy sample obtained 12 years ago. As a result, we detected fibrinoid necrosis, and the neutrophilic infiltration of small and medium sized arteries without capillary involvement, which was suggestive of PAN. Postoperative coronary angiography revealed that the internal mammary artery graft was patent, and no stenotic changes were detected (Fig. 2). Although the patient's vasculitis was considered to be inactive, the oral administration of azathioprine $(2 \mathrm{mg} / \mathrm{kg} /$ day $)$ was considered as a prophylactic immunosuppressive therapy, but the patient refused to receive immunosuppressive treatment. Therefore, she was given anti-platelet (100 mg/day aspirin) and anticoagulation therapy ( $3 \mathrm{mg} /$ day Coumadin) during the follow-up period. Fortunately, the patient did not develop the major symptoms of vasculitis, and her biological findings (C-reactive protein level and erythrocyte sedimentation rate) remained normal. We followed-up the coronary artery bypass graft by computed tomography 


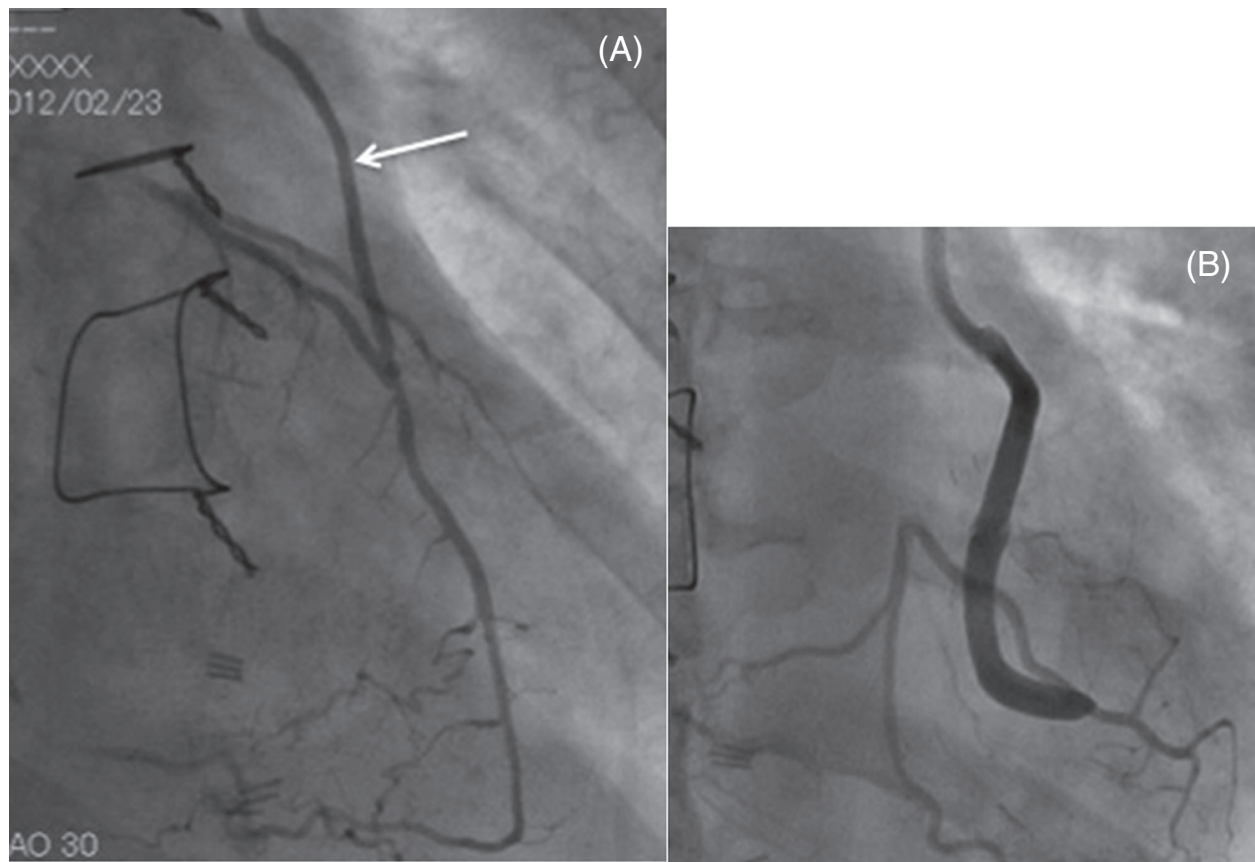

Fig. 2 Postoperative coronary angiography showing a patent internal mammary artery (IMA) graft with no stenosis or aneurysmal changes (A, arrow). A saphenous vein graft also provided good blood flow to the circumflex system $(\mathbf{B})$.
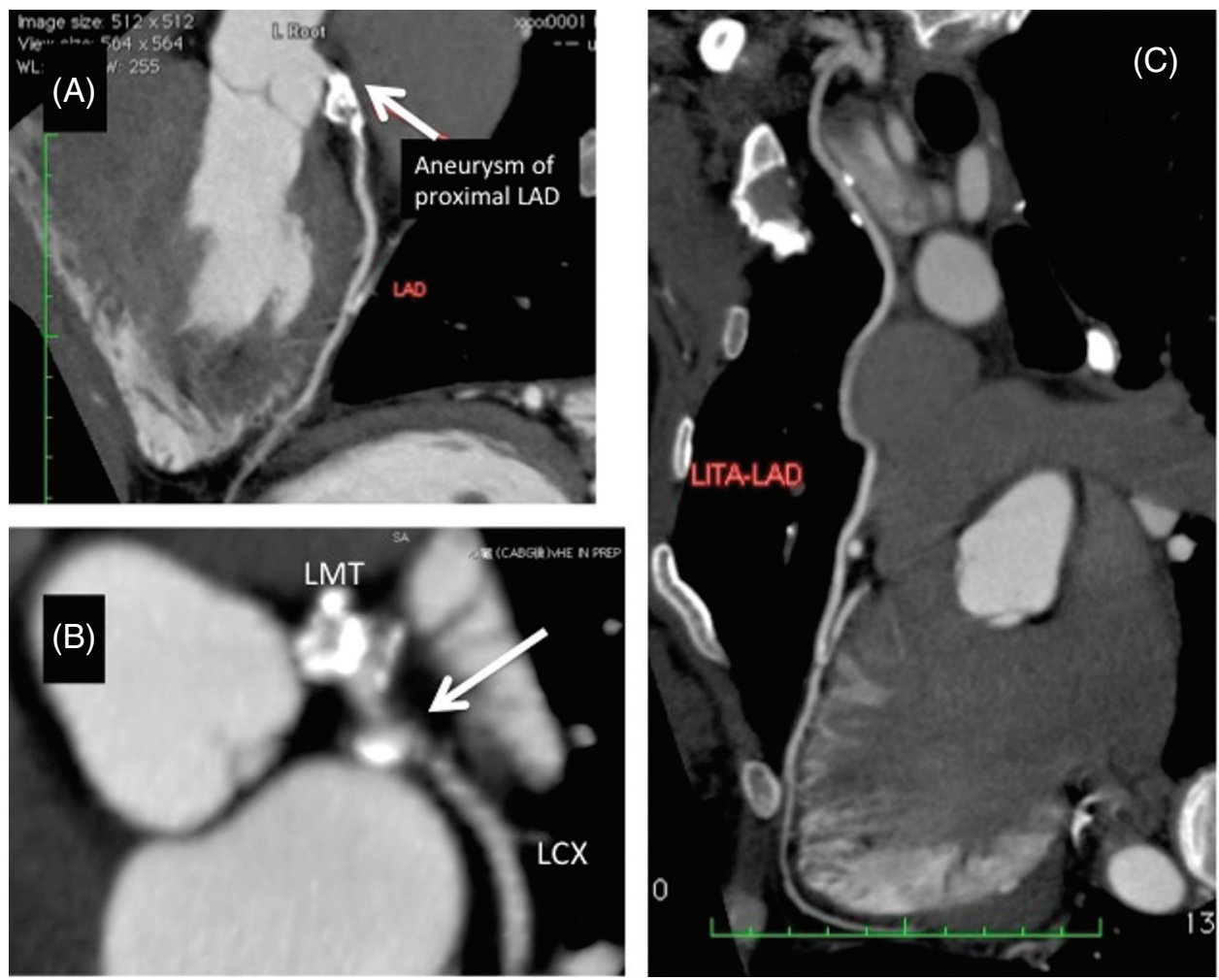

Fig. 3 Follow-up computed tomography angiography (CTA) performed 14 months after the operation. Aneurysms of proximal left anterior descending coronary artery (A, arrow), and circumflex system (B, arrow) are still present, without progression of coronary artery disease in the distal lesion. Internal mammary artery is patent with no calcification or aneurysmal changes $(\mathbf{C})$. 
angiography (CTA) at 14 postoperative months. As a result, we found that the internal mammary artery graft displayed good patency, and the coronary artery aneurysm had not progressed (Fig. 3).

\section{Discussion}

In previous clinical and necropsy series including 283 patients, congestive heart failure (27\%) and hypertension (37\%) were found to be common cardiac presentations of PAN, whereas myocardial infarction was rare $(2 \%){ }^{3)}$ PAN presenting with acute myocardial infarction and complicated by multiple coronary aneurysms is also very rare, and only 3 such cases have been reported. ${ }^{4)}$ In addition, this is the first case in which coronary artery bypass grafting was used for myocardial infarction and multiple coronary aneurysms.

There are two reports about coronary artery bypass grafting for PAN related to ischemic heart disease, and the optimal choice of bypass graft for PAN is unknown. There has been one report in which coronary artery bypass for arterial PAN was performed using saphenous vein grafts alone. ${ }^{5)}$ However, another author used LIMA grafts to revascularize the left anterior descending coronary artery because their vasculitis had been medically controlled at the time of surgery, and the patient exhibited a good postoperative course. ${ }^{6}$ In our case, the patient's C-reactive protein level and erythrocyte sedimentation rate remained normal, which were suggestive of low vasculitis activity; thus, we attempted to use a LIMA graft. Since we did not conduct any preoperative angiographic evaluations of the LIMA, because the grafting procedure was performed emergently, we evaluated the condition of the LIMA graft intraoperatively. The LIMA was in good condition and did not display any aneurysmal changes. Harvesting the LIMA graft with the skeletonization technique and an ultrasound scalpel helped us to macroscopically evaluate whether the graft was suitable. As the free flow of the LIMA graft was promising, we decided to use it to revascularize the left anterior descending coronary artery.

In Takayasu arteritis, the aorta and large vessels including the subclavian arteries are mainly affected; thus, internal mammary artery (IMA) are not ideal graft materials because of the risk of subclavian artery stenosis and occlusion. ${ }^{7)}$ However, subclavian artery stenosis is rarely reported in PAN, and there is little data about vasculitis in IMA.
There is only one report with the angiography of upper extremity, which showed occlusion and multiple aneurysmal changes of distal axillary artery and brachial artery in PAN ${ }^{8}{ }^{8}$ But, they did not mention the subclavian artery stenosis and other branches such as internal mammary artery or vertebral artery.

Further long-term follow-up is required to determine the optimal graft design for coronary artery bypass grafting in this patient population. An interesting finding from the present case was the occurrence of myocardial infarction in PAN when the patient's systemic inflammation and vasculitis seemed to be relatively inactive. This case indicates that coronary artery involvement should be carefully monitored during the chronic phase of PAN.

\section{Disclosure Statement}

None of the authors has any financial or other potential conflicts of interest.

\section{References}

1) Watts RA, Jolliffe VA, Carruthers DM, et al. Effect of classification on the incidence of polyarteritis nodosa and microscopic polyangiitis. Arteritis Rheum 1996; 39: 1208-12.

2) Guillevin L, Lhote F, Cohen P, et al. Polyarteritis nodosa related to hepatitis B virus. A prospective study with long-term observation of 41 patients. Medicine (Baltimore) 1995; 74: 238-53.

3) Cassling RS, Lortz JB, Olson DR, et al. Fatal vasculitis (periarteritis nodosa) of the coronary arteries: angiographic ambiguities and absence of aneurysms at autopsy. J Am Coll Cardiol 1985; 6: 707-14.

4) Kastner D, Gaffney M, Tak T. Polyarteritis nodosa and myocardial infarction. Can J Cardiol 2000; 16: 515-8.

5) Uçar HI, Oç M, Tok M, et al. Coronary artery bypass with saphenous vein graft in a middle-aged patient with polyarteritis nodosa. Anadolu Kardiyol Derg 2007; 7: 231-2.

6) Yanagawa B, Kumar P, Tsuneyoshi H, et al. Coronary artery bypass in the context of polyarteritis nodosa. Ann Thorac Surg 2010; 89: 623-5.

7) Amano J, Suzuki A. Surgical treatment of cardiac involvement in Takayasu arteritis. Heart Vessels Suppl 1992; 7: 168-78.

8) Chung DC, Choi JE, Song YK, et al. Polyarteritis nodosa complicated by chronic total occlusion accompanying aneurysms on all coronary arteries. Korean Circ J 2012; 42: 568-70. 